\title{
Popeye domain-containing 1 is down-regulated in failing human hearts
}

\author{
RACHEL GINGOLD-BELFER $^{1,2}$, MICHAEL BERGMAN $^{1,2}$, YIFAT ALCALAY $^{1}$, HADASSA SCHLESINGER $^{1}$, \\ DAN ARAVOT ${ }^{3,5}$, MARIUS BERMAN 3,6 , HERTZEL SALMAN ${ }^{2}$, \\ THOMAS BRAND ${ }^{4,7}$ and GANIA KESSLER-ICEKSON ${ }^{1}$ \\ ${ }^{1}$ Basil and Gerald Felsenstein Medical Research Center, Sackler Faculty of Medicine, Tel-Aviv University; \\ Departments of ${ }^{2}$ Internal Medicine C, Golda Campus and ${ }^{3}$ Cardiothoracic Surgery, Beilinson Campus, \\ Rabin Medical Center, Petach-Tikva, Israel; ${ }^{4}$ Department of Cell and Developmental Biology, \\ University of Wuerzburg, Wuerzburg, Germany
}

Received August 3, 2010; Accepted October 3, 2010

DOI: $10.3892 / \mathrm{ijmm} .2010 .558$

\begin{abstract}
Congestive heart failure, a complex disease of heterogeneous etiology, involves alterations in the expression of multiple genes. The Popeye domain-containing (POPDC) family of three novel muscle-restricted genes (POPDC1-3) is evolutionarily conserved and developmentally regulated. In mice, POPDC1 has been shown to play an important role in skeletal and cardiac muscles subjected to injury or stress. However, it has never been explored in human hearts. In biopsies from non-failing and failing human hearts, we examined the cellular distribution of POPDC1 as well as the expression patterns of POPDC1-3 mRNAs. POPDC1 was visualized by immunohistochemistry and estimated by Western immunoblotting. The mRNA levels of POPDC1-3 and $B$ myosin heavy chain (MYHC7) were assessed using reverse transcription/quantitative polymerase chain reaction. POPDC1 was predominantly localized in the sarcolemma with an enhanced expression in the intercalated discs. In failing hearts, many cardiomyocytes appeared deformed and POPDC1 labeling was deranged. The three POPDC mRNAs
\end{abstract}

Correspondence to: Dr Gania Kessler-Icekson, Basil and Gerald Felsenstein Medical Research Center, Sackler Faculty of Medicine, Tal-Aviv University, Beilinson Campus, Rabin Medical Center, Petach-Tikva 49100, Israel

E-mail: icekson@post.tau.ac.il

Present addresses: ${ }^{5}$ Department of Cardiothoracic Surgery, Carmel Hospital, Haifa, Israel; ${ }^{6}$ Department of Cardiothoracic Surgery, Papworth Hospital, Cambridge; ${ }^{7}$ Heart Science Centre, Imperial College, London, UK

Key words: Popeye domain-containing genes 1-3, gene expression, immunohistochemistry were expressed in the four heart chambers with higher transcript levels in the ventricles compared to the atria. Heart failure concurred with reduced levels of POPDC1 mRNA and protein in the left ventricle. Correlation analyses of mRNA levels among the failing heart specimens indicated the coordinated regulation of $P O P D C 1$ with $P O P D C 3$ and of $P O P D C 2$ with $M Y H C 7$. It can be concluded that POPDC gene expression is modified in end-stage heart failure in humans in a manner suggesting regulatory and/or functional differences between the three family members and that POPDC1 is particularly susceptible to this condition.

\section{Introduction}

Heart failure, a complex disease of heterogeneous etiologies and increasing morbidity rates (1), is associated with alterations in the expression of multiple myocardial genes (2). The exact role played by many of these genes in heart failure is not always understood, as is the relevance to heart failure of recently identified novel myocardial genes.

The Popeye domain-containing (POPDC) genes represent an example of a novel family of highly conserved and developmentally regulated muscle-restricted genes. In vertebrates, three family members, $P O P D C 1-3$, have been known that encode transmembrane proteins, the prototypic structure of which includes a glycosylated extracellular amino-terminus, three transmembrane helices and a conserved intracellular Popeye domain that contains a homodimerization motif (3-6). POPDC1, also known as blood vessel epicardial substance (BVES), has been identified in eukaryotes from insects to man, whereas POPDC2 and 3 have only been found in higher vertebrates $(3,6-8)$. POPDC1 has been proposed to play a role in cell adhesion and cell signaling as it has been found to be localized at sites of intercellular contacts, bound to the signaling modifier guanine nucleotide exchange factor $\mathrm{T}$, and has been shown to be involved in Rho signaling and receptor cycling (8-13). Experiments using Popdc1-null mice have indicated an impaired ability for skeletal muscle regeneration (14) and an increased sensitivity of the heart to ischemia-reperfusion 
injury (Alcalay et al, unpublished data). We have recently identified POPDC1 in the caveolae (membrane microdomains that regulate cytoprotection) of mouse heart myocytes that could explain its role in ischemic tolerance (Alcalay et al, unpublished data).

In light of the possibility that POPDC1 plays a role in the heart during damage or stress, we examined the supposition that POPDC gene expression could be altered in heart dysfunction or failure. In failing and non-failing human hearts, we characterized the cellular distribution of POPDC1, assessed its expression levels and questioned whether the expression of the POPDC family members is coordinately regulated.

\section{Materials and methods}

Patients and tissues. The Institutional Review Board approved the study (approval no. 2640) and informed consent was obtained from each subject or their guardian. The hearts of 12 patients undergoing cardiac transplantation for chronic end-stage heart failure $(F)$ and 2 controls ( $C$, no matched recipients), were recruited. The $\mathrm{F}$ group patients were in the New York Heart Association functional classes III and IV (Table I). Biopsies of the four heart chambers were obtained from four failing hearts and the control hearts. In all the other hearts only the left ventricle (LV) was examined. Upon removal, the biopsies were coded, snap-frozen in liquid nitrogen and stored at $-70^{\circ} \mathrm{C}$. After the molecular analysis was completed anonymously, the specimens were decoded and their clinical background was unmasked.

Immunohistochemistry. Samples of the frozen tissues were embedded in Tissue-Tek (Miles Diagnostics Division, USA) and $5-8-\mu \mathrm{m}$-thick cryosections were cut out (2800 Frigocut, Reichert-Jung, Germany), and were paraformaldehyde-fixed (4\% in PBS, $10 \mathrm{~min}$ ), and PBS-rinsed. Blocking in PBS containing $0.2 \% \mathrm{BSA}$ and $0.1 \%$ Triton $\mathrm{X}-100$ ( $1 \mathrm{~h}$, room temperature) was followed by incubation with the primary antibodies (overnight, $4^{\circ} \mathrm{C}$ ), PBS rinse (x3) and then incubation with the secondary antibodies (1 h, room temperature). The primary antibodies were polyclonal goat anti-BVES/ POPDC1 (1:50), rabbit anti-connexin 43 (1:100) (Santa Cruz, CA, USA), and mouse anti $\alpha$-actinin (1:500) (Sigma, MO, USA). The secondary antibodies were Cy3-donkey anti-goat (1:100), DyLight 649-donkey anti-rabbit $(1: 200)$ and DyLight 488-donkey anti-mouse polyclonal antibodies (Jackson ImmunoResearch Laboratory, West Grove, PA, USA). The nuclei were counter-labeled by Hoechst (Sigma). Visualization and photography were performed using Leica TCS SP5 Confocal Imaging Systems and x63 oil objectives.

Western blot analysis. A tissue sample was homogenized and the proteins were subjected to SDS-PAGE and Western immunoblotting as previously described, loading $10 \mu \mathrm{g}$ protein per lane (16). POPDC1 was detected by the above anti-BVES antibodies (diluted 1:200) and HRP-bovine antigoat IgG (Santa Cruz). Loading was corrected for actin, using mouse anti-actin monoclonal antibody (Santa Cruz) and HRP-rabbit anti-mouse IgG (Jackson ImmunoResearch). Reactive bands were quantified using the VersaDoc Imaging System (BioRad, CA, USA).
RNA preparation and analysis. Total RNA was isolated (Trireagent, Sigma) and subjected to reverse transcription and quantitative polymerase chain reaction (RT-qPCR). Firststrand cDNA was synthesized in $20 \mu 1$ reactions containing $1 \mu \mathrm{g}$ total RNA, $100 \mathrm{U}$ M-MLVRT (H-) reverse transcriptase (Promega, Madison, WI, USA) and $10 \mu \mathrm{M}$ oligo(dT) ${ }_{12-18}$ (17). Aliquots (0.02-1.5 $\mu \mathrm{l})$ of each cDNA were taken for qPCR analysis (TaqMan technology, Applied Biosystems, Foster City, CA, USA) performed in the Prism 7700 Sequence Detection System. The following Assays-on-Demand (Applied Biosystems) were used: i) POPDC1, Hs00362584_M1, ii) POPDC2, Hs02379301_S1, iii) POPDC3, Hs00223577_M1, and iv) MYHC7, Hs01110632_M1. In every specimen, the results obtained for each of the mRNAs were normalized to the human RPLP0 ribosomal protein mRNA. The results, expressed in relative quantity values, were further normalized to the values obtained from the $\mathrm{LV}$ of explant $\mathrm{C} 1$ that was considered as 1 . The mRNA scores are presented in arbitrary units.

Statistics. Comparison between the groups was carried out by the Student's t-test. Pearson's correlation analysis was employed to test the relationship between the gene expression levels. A value of $\mathrm{P}<0.05$ was considered to be statistically significant.

\section{Results}

POPDC1 localization in cardiomyocytes. In the normal tissues, POPDC1 appeared both in the lateral sarcolemma and in the intercalated discs of cardiomyocytes and within the intercalated discs, it was co-localized with the gap junction protein, connexin 43 (Cx43) (Fig. 1A). Intracellular POPDC1 immunolabeling was also noted. In the failing hearts, the distribution of both POPDC1 and Cx43 was disturbed, as were cardiomyocyte morphology and cross-striations (Fig. 1B). The failing cardiomyocytes were larger, their cross-striations were irregular, fewer intercalated discs were evident and Cx43 was also found in the lateral sarcolemma. POPDC1 labeling was correlated with the derangement in cell morphology and the labeling intensity in the intercalated discs was not as prominent as in the non-failing tissues. Similar immunohistological results were obtained in several failing heart specimens (F6, F7, F8 and F9).

POPDC gene expression in the heart chambers. As shown in Fig. 2, all three POPDC genes were expressed in the four heart chambers and in the control hearts, the expression levels were higher in the ventricles than in the atria (the other control, C2, resembled C1). When compared to the control hearts, a reduction in expression levels was evident in the failing hearts, particularly in the case of POPDC1 and 3 mRNAs, and the alterations varied among the specimens.

POPDC1 expression is lower in failing hearts. In the LV specimens, an association of heart failure with reduced POPDC1 expression was observed. However, the degree of this reduction varied among the individuals (Fig. 3A). In certain patients, such as F1 (IDC) or F11 (IHD), the levels of POPDC 1 mRNA decreased to $30-40 \%$ of the controls, 
Table I. Clinical details of the patients.

\begin{tabular}{|c|c|c|c|c|c|}
\hline Patient & Age, years & Gender & Diagnosis & $\mathrm{EF}(\%)$ & Other clinical background \\
\hline $\mathrm{F} 1$ & 55 & M & IDC & $<20$ & Anemia, smoking \\
\hline $\mathrm{F} 2$ & 23 & M & IDC & $<20$ & Epilepsy, smoking \\
\hline F3 & 66 & M & IHD & 12 & Atrial fibrillation \\
\hline $\mathrm{F} 4$ & 53 & M & IHD & 11 & Hypertension, COPD, hyperlipidemia \\
\hline F5 & 54 & M & IDC & 28 & Diabetes, COPD, hyperlipidemia, smoking \\
\hline F6 & 6 & M & $\mathrm{CDC}$ & 16 & Hypertension \\
\hline F7 & 51 & M & IHD & 7 & Chest radiation as lymphoma treatment \\
\hline F8 & 59 & M & IHD & 13 & Smoking \\
\hline F9 & 50 & $\mathrm{M}$ & IHD & 26 & Chronic renal failure, stroke, smoking \\
\hline F10 & 51 & M & IHD & 15 & Smoking \\
\hline F11 & 63 & M & IHD & $<20$ & Schizophrenia, smoking \\
\hline F12 & 60 & M & IHD & $<20$ & Smoking \\
\hline C1 & 32 & $\mathrm{Fe}$ & $\mathrm{C}$ & & \\
\hline $\mathrm{C} 2$ & 5 & M & $\mathrm{C}$ & & \\
\hline
\end{tabular}

F, chronic end-stage heart failure; C, control; EF, ejection fraction; IDC, idiopathic dilated cardiomyopathy; IHD, ischemic heart disease; $\mathrm{CDC}$, congenital dilated cardiomyopathy; $\mathrm{M}$, male; $\mathrm{Fe}$, female.

A
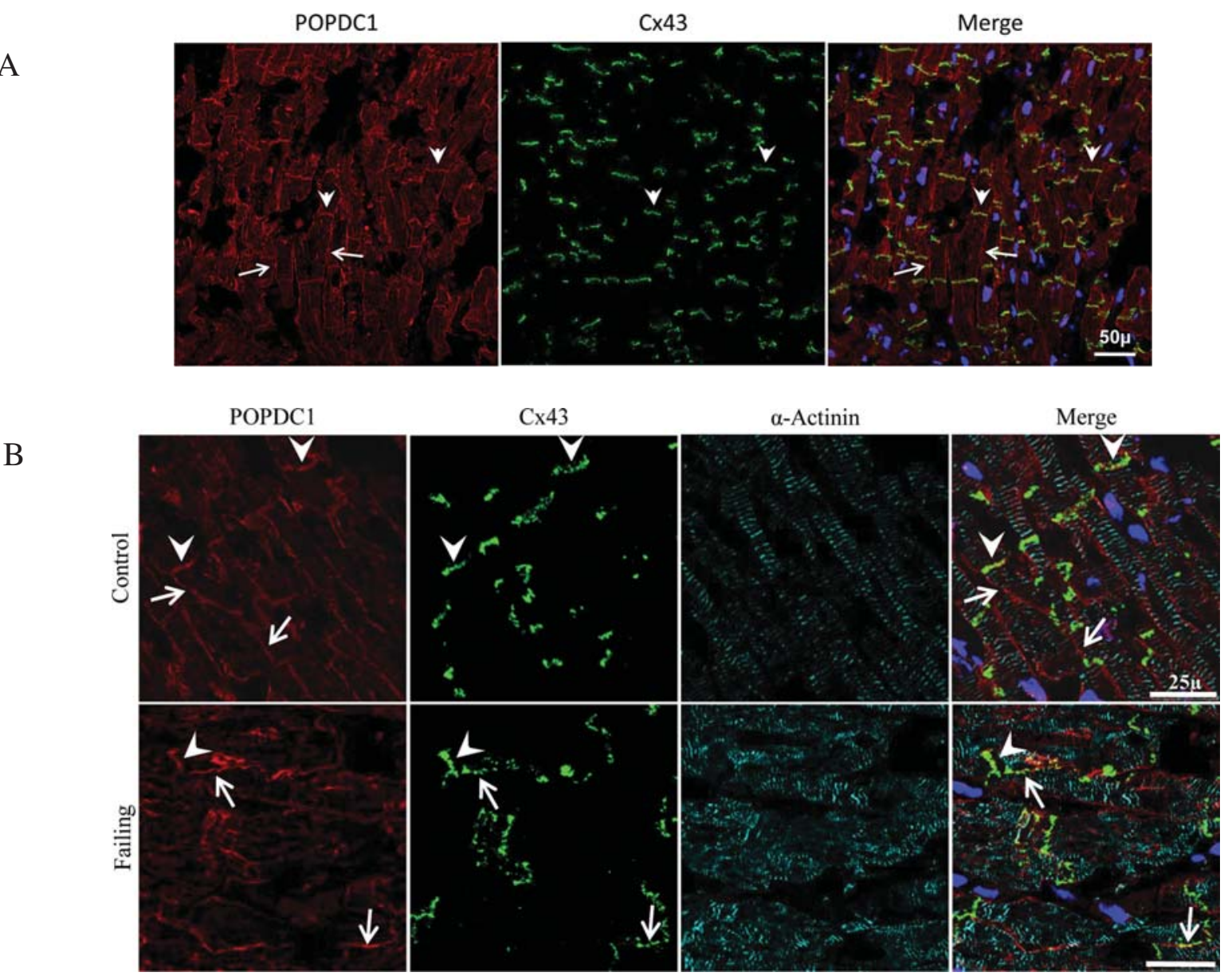

Figure 1. POPDC1 cellular localization. (A) A representative cryosection from the LV of a control heart (C1) that was co-labeled with antibodies directed against POPDC1 (red) and Cx43 (green). Nuclei were counter-stained with Hoechst (blue). Merge, co-localization of POPDC1 and Cx43 (yellow) in the intercalated discs. Arrows, lateral sarcolemma; arrowheads, intercalated discs. POPDC1 is also noticeable inside the cells. (B) Cryosections from normal (C1) and failing (F7) LV viewed at a higher magnification. Co-immunolabeling of POPDC1 (red), Cx43 (green), and $\alpha$-actinin (light blue). In the normal heart the intercalated discs (arrowheads) align with the cross-striations ( $\alpha$-actinin). In the end-stage failing heart the cardiomyocytes appear deformed, apparently enlarged, their cross striations are disarrayed, and in the intercalated discs, POPDC1 is less evident. Cx43 is also distributed in the lateral sarcolemma (arrows), perpendicular to the cross-striations and intercalated discs. 


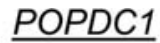

C1

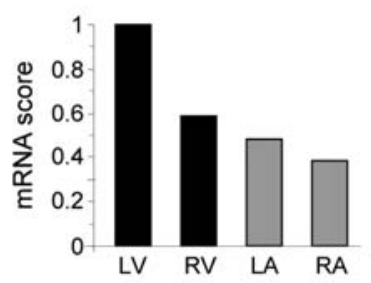

F7

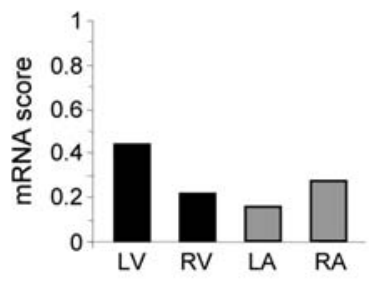

F10

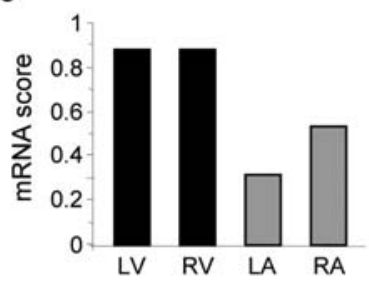

F11

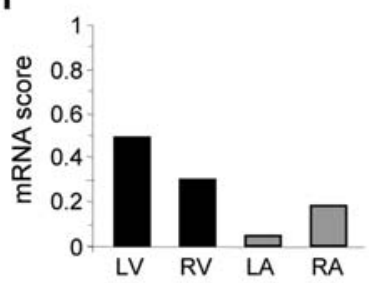

F12

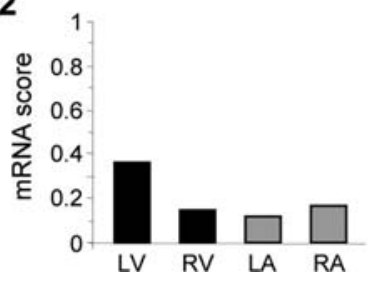

$\underline{P O P D C 2}$
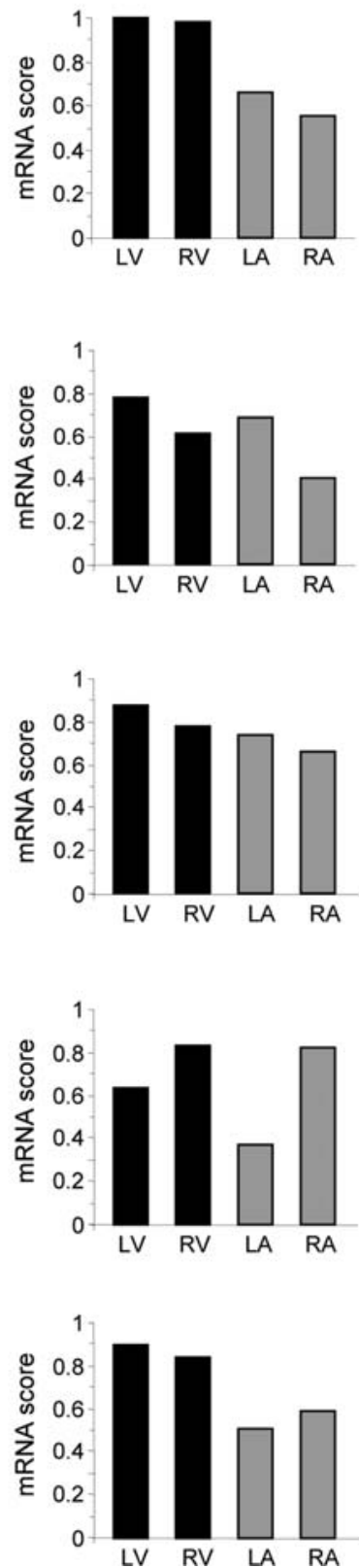
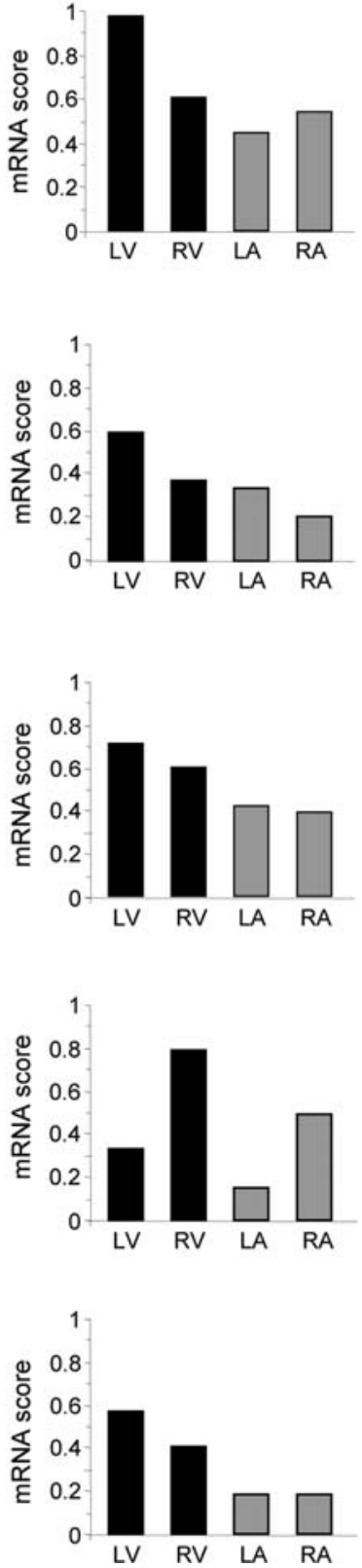

Figure 2. POPDC transcript distribution in the heart chambers. Total RNA obtained from the left and right ventricles (LV, RV) and atria (LA, RA) was assessed by RT-qPCR. Shown are a non-failing (C1) and failing (F7, F10, F11, F12) hearts. The results of each POPDC mRNA were normalized to those in the $\mathrm{LV}$ of specimen $\mathrm{C} 1$ that was considered as 1.

whereas in others, such as F10 (IHD) or F6 (IHD), these levels remained as high as 70-80\% compared to the controls. No distinction could be established between the IHD and idiopathic dilated cardiomyopathy (IDC) hearts. The expression levels of POPDC 2 and 3 in certain specimens were low, while their expression levels in other specimens resembled the control hearts (data not shown). In agreement with the transcript analysis, Western blot evaluation of POPDC1 protein levels demonstrated reduced amounts of the protein in the failing hearts compared to the non-failing controls as well as noticeable variations between individuals (Fig. 3B).
Coordinated regulation of POPDC gene expression. In order to test whether the three POPDC genes were coordinately regulated in failing hearts, we conducted correlation analyses between the mRNA levels of the three POPDC transcripts within the group of the failing LV samples. While no correlation was observed between the mRNA levels of POPDC2 and POPDC1 (r=0.106) or POPDC3 and POPDC2 $(\mathrm{r}=0.116)$ (data not shown), a clear correlation was found between POPDC3 and 1 expression $(\mathrm{r}=0.679, \mathrm{P}=0.015)$, suggesting the coordinated regulation of these two POPDC family members (Fig. 4A). In addition, we examined the possibility that the diminution in POPDC mRNA corresponds 

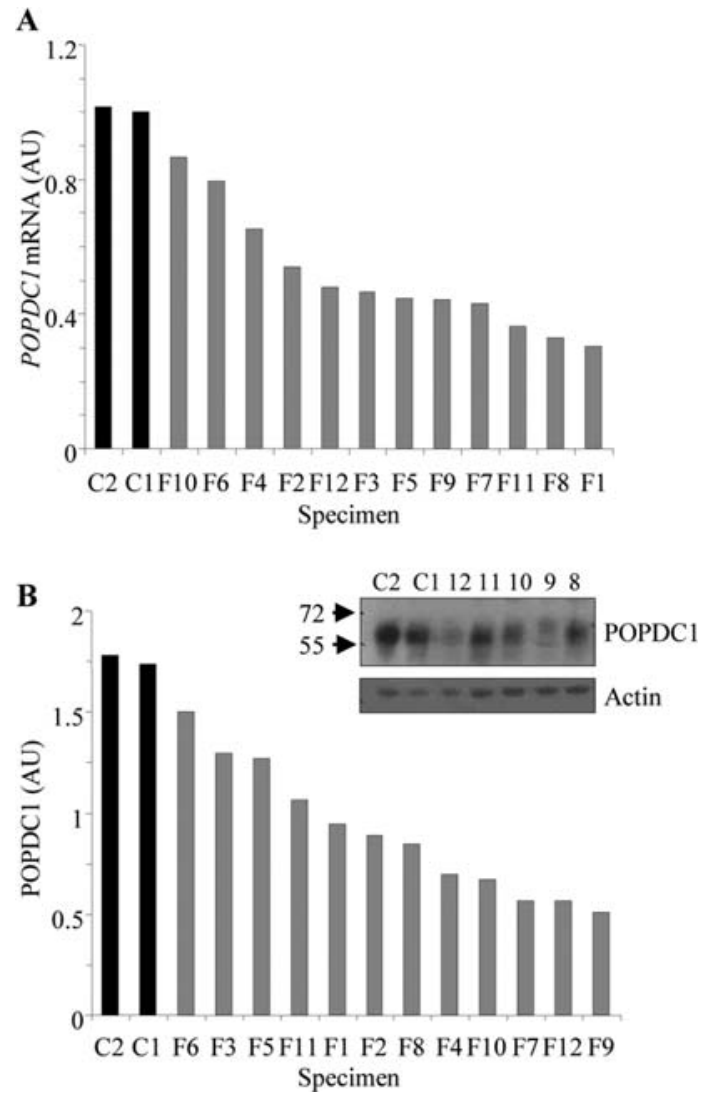

Figure 3. Reduced POPDC1 expression in failing left ventricles. Expression levels of POPDC1 mRNA (A) and protein (B) in the LV of non-failing (black) and failing (gray) hearts presented in descending order. The inset in (B) depicts immunoblots of representative specimens including the two nonfailing $(\mathrm{C} 1, \mathrm{C} 2)$ and five failing (F12, F11, F10, F9 and F8) hearts. Arrows indicate 55 and $72 \mathrm{kDa}$ molecular weight markers. POPDC1 migrates at $\sim 60 \mathrm{kDa}$. AU, arbitrary units.

A

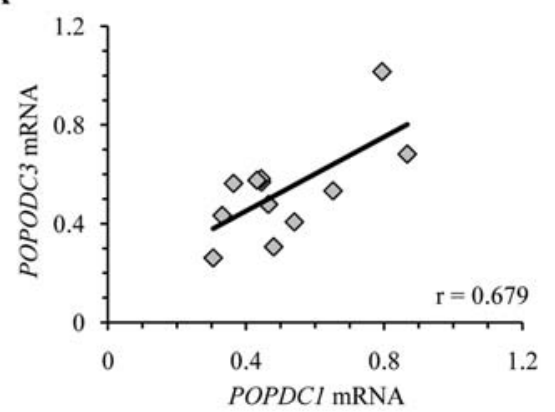

B

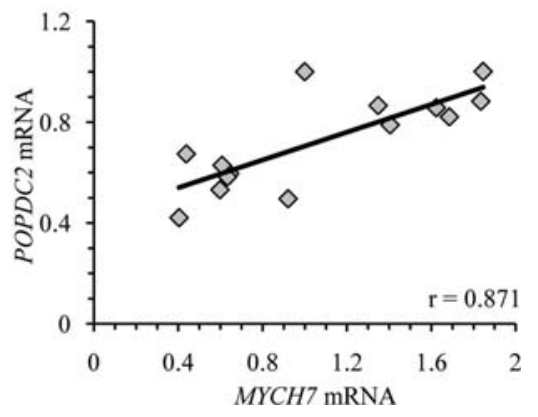

Figure 4. Scatter plots showing correlation co-efficients between the mRNA levels of (A) POPDC 3 and POPDC1, (B) POPDC2 and $M Y H C 7$, within the failing hearts group; $\mathrm{N}=12$. to a general down-regulation of cardiomyocyte structural proteins. We examined the relationship between the POPDC1-3 and MYHC7 mRNA levels. While no correlation was observed between the MYHC7 and the POPDC1 and 3 mRNAs, a significant correlation coefficient was found between the POPDC2 and MYHC 7 mRNAs (r=0.871, $\mathrm{P}=0.0002$ ), suggesting a similar pattern of regulation of these two genes (Fig. 4B).

\section{Discussion}

Previous studies have indicated a role for POPDC1 in the pathophysiology of cardiac and skeletal muscles (14). Our observations that the hearts of transgenic mice lacking POPDC1 manifest impaired recovery from ischemiareperfusion injury, along with our recent finding of POPDC1 in the caveolae of cardiomyocytes (Alaclay et al, unpublished data), as well as the suggestion that POPDC1 plays a role in cell signaling and receptor cycling $(10,12,13)$, advocate that POPDC1 is important for heart function.

Our study is the first demonstration of POPDC1 membrane-localization within the human heart. In the intercalated discs it is co-localized with Cx43. In this respect, it resembles the mouse heart in which POPDC1 was also conspicuous in the intercalated discs (9). The intercalated discs are membrane domains that provide physical linkage and facilitate electrical signal propagation between neighboring cardiomyocytes, maintaining the proper contractility and rhythmicity of the myocardium (18). Heart failure is associated with myocyte disarray and alterations in the content and distribution of many proteins, changes that explain the impairment of myocardial mechanical and electrical functions in this condition $(15,19,20)$. Studies have described a reduction in $\mathrm{Cx} 43$ content and its redistribution to the lateral sarcolemma in end-stage heart failure in humans and a correlation with heart malfunction has been proposed (20). We noted a diminution in the intensity of POPDC1 labeling in the intercalated discs relative to the lateral sarcolemma, as well as the appearance of $\mathrm{Cx} 43$ in the latter. However, the decrease in POPDC1 immunolabeling was not as evident as the reduction observed by Western blot analysis. Possible explanations could be that the overall reduction in POPDC1 is due to cardiomyocyte death, which is common in heart failure, and not to a significant reduction in POPDC1 content per cell, or that immunohistochemistry is not sensitive enough to depict a partial reduction in the amount of a protein.

Finding the three POPDC transcripts in the four heart chambers was not unexpected as they have been found in developing hearts by means of in situ hybridization (3). Our quantitative evaluation provides new information as it identifies greater POPDC expression in the ventricles compared to the atria, which could correspond to functional or structural differences between the heart compartments. Compartment-dependent differences have been reported for myoglobin (21), adenosine A1 receptor (22) and the phospholamban-to-SERCA2 ratio (23), the respective regulators of intramuscular oxygen tension, adenosine responsive pathways, and the rates of force development and relaxation. In the failing hearts we detected a clear reduction 
in left ventricular POPDC1 mRNA that could possibly lead to the diminution of the protein synthesis and content. Diminution in cardiac specific proteins and mRNAs in endstage failing human hearts has been shown for myosin, titin, Cx43, angiotensin II receptors and other proteins and has been related to muscle cell degeneration and malfunction $(20,23,24)$. We could not establish a relationship between the level of POPDC1 expression and the degree of malfunction or the etiology of heart failure (IHD or IDC). Such miscorrelation has been reported in explanted hearts and has been attributed to the severe pathological condition and the extremely low performance of the tissues analyzed, common to end-stage heart failure rather than to any specific etiology (25).

We also observed a similar reduction in POPDC1 mRNA and protein in experimental acute ischemia-reperfusion injury in the mouse (Alcalay et al, unpublished data). As POPDC1 is a protein of the caveolae and as Popdc1-null hearts have a reduced caveolae number and exhibit higher vulnerability to ischemia-reperfusion (Alcalay et al, unpublished data), we believe that the reduction in POPDC1 levels is not a mere outcome of the degenerative condition but likely contributes to heart deterioration.

To date, no detailed information is available concerning the regulation of POPDC1-3 expression. The post transcriptional regulation of POPDC1 during mouse post-natal development and the POPDC1 down-regulation by serum in cultured cardiomyocytes have been proposed, although no clear mechanism has been delineated $(14,17)$. In the failing hearts we found the co-regulation of $P O P D C 1$ with $P O P D C 3$ but not with $P O P D C 2$, which correlated with $M Y H C 7$. $P O P D C 1$ and $P O P D C 3$ are organized in tandem at chromosomal locus $6 \mathrm{q} 21$ and the co-regulation of the two cannot be ruled out (5). As it is generally accepted that genes demonstrating orchestrated expression relate to the same biological process or condition, the co-regulation of $P O P D C 2$ and $M Y H C 7$ could indicate the functional or structural relationship of the two gene products in the failing heart.

The investigation of molecular mechanisms underlying human heart function and disease is difficult, as human heart tissues are not easily accessible for sampling or experimental manipulation. Thus, a limitation to this study was the restricted number of human samples, particularly the small number of controls. In this study, the patient age ranged from 5 to 66 years. The controls included a 6-year-old child and a 32-yearold adult. We considered these controls as valid as we had a 5year-old child among our diseased patients and as previous reports have not indicated major differences in heart gene expression between the ages we were studying $(15,20,25,27)$. Overall, myocardial biopsies removed during heart surgery have enabled the profiling of the human myocardium molecular phenotype $(18,22-27)$, and although descriptive, these results have greatly advanced our understanding of maladaptive and pathological changes in the human heart. Our study also provides new information as to the molecular database of the human myocardium and expands our knowledge of POPDC 1 and the POPDC genes, which together contribute to the comprehension of the heart failure condition.

\section{Acknowledgements}

This study was supported by the German-Israeli Foundation (G.K.-I. and T.B.), the Israel Science Foundation (G.K.-I.), the Hammer-Fingerhut Fund, Tel-Aviv University (G.K.-I. and M.B.), and the Rabin Medical Center Fund for Young Physicians (R.G.-B.).

\section{References}

1. Cowie MR, Mosters A, Wood DA, et al: The epidemiology of heart failure. Eur Heart J 18: 208-225, 1997.

2. Liew CC and Dzau VJ: Molecular genetics and genomics of heart failure. Nat Rev Genet 45: 811-825, 2004.

3. Andree B, Hillemann T, Kessler-Icekson G, et al: Isolation and characterization of the novel Popeye gene family expressed in skeletal muscle and heart. Dev Biol 223: 371-382, 2000.

4. Reese DE and Bader DM: Cloning and expression of hbves, a novel and highly conserved mRNA expressed in the developing and adult heart and skeletal muscle in the human. Mamm Genome 10: 913-915, 1999.

5. Andree B, Fleige A, Hillemann T, Arnold HH, Kessler-Icekson G, and Brand T: Molecular and functional analysis of Popeye genes. A novel family of transmembrane proteins preferentially expressed in heart and skeletal muscle. Exp Clin Cardiol 7: 99-103, 2002.

6. Brand T: The Popeye domain-containing gene family. Cell Biochem Biophys 43: 95-103, 2005.

7. Osler ME, Smith TK and Bader DM: Bves, a member of the Popeye domain-containing gene family. Dev Dyn 235: 586-593, 2006.

8. Hager HA and Bader DM: Bves: Ten years after. Histol Histopathol 24: 777-787, 2009.

9. Smith TK and Bader DM: Characterization of Bves expression during mouse development using newly generated immunoreagents. Dev Dyn 235: 1701-1708, 2006.

10. Smith TK, Hager HA, Francis R, Kilkenny DM, Lo CW and Bader DM: Bves directly interacts with GEFT, and controls cell shape and movement through regulation of $\mathrm{Rac} 1 / \mathrm{Cdc} 42$ activity. Proc Natl Acad Sci USA 105: 8298-8303, 2008.

11. Kawaguchi M, Hager HA, Wada A, Koyama T, Chang MS and Bader DM: Identification of a novel intracellular interaction domain essential for Bves function. PLoS One 3: e2261, 2008.

12. Russ PK, Kupperman AI, Presley SH, Haselton FR and Chang MS: Inhibition of RhoA signaling with increased Bves in trabecular meshwork cells. Invest Ophthalmol Vis Sci 51: 223-230, 2010.

13. Hager HA, Roberts RJ, Cross EE, Proux-Gillardeaux V and Bader DM: Identification of a novel Bves function: regulation of vesicular transport. EMBO J 29: 532-545, 2010.

14. Andree B, Fleige A, Arnold HH and Brand T: Mouse Pop1 is required for muscle regeneration in adult skeletal muscle. Mol Cell Biol 22: 1504-1512, 2002.

15. Hein S, Scholz D, Fujitani N, et al: Altered expression of titin and contractile proteins in failing human myocardium. J Mol Cell Cardiol 26: 1291-1306, 1994.

16. Kessler-Icekson G, Schlesinger H, Freimann S and Kessler E: Expression of procollagen $\mathrm{C}$-proteinase enhancer-1 in the remodeling rat heart is stimulated by aldosterone. Int $\mathrm{J}$ Biochem Cell Biol 38: 358-365, 2006.

17. Parnes D, Jacoby V, Sharabi A, Schlesinger H, Brand T and Kessler-Icekson G: The POPDC gene family in the rat: Molecular cloning, characterization and expression analysis in the heart and cultured cardiomyocytes. Biochim Biophys Acta 1769: 586-592, 2007.

18. Noormana M, van der Heydena MA, van Veena TA, et al: Cardiac cell-cell junctions in health and disease: Electrical versus mechanical coupling. J Mol Cell Cardiol 47: 23-31, 2009.

19. Arai M, Alpert NR, MacLennan DH, Barton P and Periasamy M: Alterations in sarcoplasmic reticulum gene expression in human heart failure. A possible mechanism for alterations in systolic and diastolic properties of the failing myocardium. Circ Res 72: 463-469, 1993.

20. Kostin S, Rieger M, Dammer S, et al: Gap junction remodeling and altered connexin43 expression in the failing human heart. Mol Cell Biochem 242: 135-144, 2003. 
21. Lin L, Sylvén C, Sotonyi P, Somogyi E, Kaijser L and Jansson E: Myoglobin content and citrate synthase activity in different parts of the normal human heart. J Appl Physiol 69: 899-901, 1990.

22. Musser B, Morgan ME, Leid M, Murray TF, Linden J and Vestal RE: Species comparison of adenosine and betaadrenoceptors in mammalian atrial and ventricular myocardium. Eur J Pharmacol 246: 105-111, 1993

23. Koss KL, Grupp IL and Kranias EG: The relative phospholamban and SERCA2 ratio: a critical determinant of myocardial contractility. Basic Res Cardiol 92 (Suppl 1): 17-24, 1997.

24. Regitz-Zagrosek V, Friedel N, Heymann A, et al: Regulation, chamber localization, and subtype distribution of angiotensin II receptors in human hearts. Circulation 91: 1461-1471, 1995.
25. Steenman M, Chen VW, le Cunff M, et al: Transcriptomal analysis of failing and nonfailing human hearts. Physiol Genomics 12: 97-112, 2003.

26. Schlesinger H, Ovil J, Levy MJ and Kessler-Icekson G: Separation and quantitation of ventricular myosin heavy chain from human atrial myocardium. Biochem Int 11: 747-753, 1985.

27. Hall JL, Birks EJ, Grindle S, et al: Molecular signature of recovery following combination left ventricular assist device (LVAD) support and pharmacologic therapy. Eur Heart J 28: 613-627, 2007. 